\title{
A SIMPLE, DIRECT PROOF OF UNIQUENESS FOR SOLUTIONS OF THE HAMILTON-JACOBI EQUATIONS OF EIKONAL TYPE
}

\author{
HITOSHI ISHII
}

\begin{abstract}
We present a new, direct proof of the uniqueness theorem for a class of Hamilton-Jacobi equations including the eikonal equation in geometric optics.
\end{abstract}

0. Introduction. This paper is devoted to the study of Hamilton-Jacobi equations of the form

$$
H(x, u, D u)=0 \quad \text { in } \Omega,
$$

where $\Omega$ is an open subset of $\mathbf{R}^{N}, H: \Omega \times \mathbf{R} \times \mathbf{R}^{N} \rightarrow \mathbf{R}, u \in C(\Omega)$, and $D u$ denotes the gradient of $u$. In the case where the Hamiltonian $H(x, u, p)$ is strictly increasing in $u$, the theory of uniqueness of a generalized solution of the Dirichlet problem for equation (0.1) has been well developed since the introduction of the notion of viscosity solution by $M$. G. Crandall and P. L. Lions [2]. We refer the reader to M. G. Crandall and P. L. Lions $[\mathbf{2}, \mathbf{3}]$ and H. Ishii $[\mathbf{5}, \mathbf{6}]$ for this development.

A typical example of Hamilton-Jacobi equations is the eikonal equation in geometric optics

$$
|D u|=n(x),
$$

where $n \in C(\bar{\Omega})$ and $n(x)>0$ on $\bar{\Omega}$. Here the Hamiltonian $H(x, u, p)=|p|-n(x)$ is independent of $u$, and so the uniqueness of a viscosity solution of the Dirichlet problem for $(0.2)$ is not a direct consequence of the uniqueness theory mentioned above. It is usually deduced from the uniqueness theory after converting equation (0.2) into equation (0.1) with a strictly increasing Hamiltonian $H(x, u, p)$ in $u$ via a transformation of the unknown (a device of S. N. Kružkov [9]). Indeed, if $u$ is a viscosity solution of $(0.2)$, then the function $v=-e^{-u}$ is a viscosity solution of $n(x) v+|D v|=0$. We refer the interested reader to Kružkov [9], and P. L. Lions [10] and [2] for the details of this approach.

The main purpose here is to present a new, direct proof of the uniqueness theorem for a class of Hamilton-Jacobi equations including (0.2) as a special case in the framework of viscosity solutions. $\S 1$ contains comparison results which yield the desired uniqueness theorem. An example of application of our comparison theorem is presented in $\S 2$. Our techniques are also useful in proving the rate of convergence of the vanishing viscosity method. This subject will be discussed in [7].

Received by the editors July 29, 1985 and, in revised form, April 7, 1986.

1980 Mathematics Subject Classification (1985 Revision). Primary 35F30; Secondary 35B05, $35 \mathrm{~L} 60$.

Key words and phrases. Hamilton-Jacobi equations, uniqueness, comparison theorems, eikonal equation. 
We do not recall here the definition and basic properties of viscosity solutions and instead refer the reader to M. G. Crandall, L. C. Evans, and P. L. Lions [1] and [2].

1. A comparison theorem. We call a function $m:[0, \infty) \rightarrow[0, \infty)$ a modulus if it is continuous, nondecreasing, and satisfies $m(0)=0$.

The following hypotheses on $H$ will be used.

(H1) The function: $u \rightarrow H(x, u, p)$ is nondecreasing on $\mathbf{R}$ for each $(x, p) \in$ $\Omega \times \mathbf{R}^{N}$.

(H2) There is a modulus $m$ such that

$$
H(y, u, \lambda(x-y))-H(x, u, \lambda(x-y)) \leq m\left(\lambda|x-y|^{2}+|x-y|\right)
$$

for $\lambda \geq 0$ and $x, y \in \Omega$.

LEMMA 1. Let $\Omega$ be a bounded open subset of $\mathbf{R}^{N}$ and $f \in C(\Omega)$ satisfy $f(x)<0$ for $x \in \Omega$. Assume (H1) and (H2) hold. Let $u, v \in C(\bar{\Omega})$ satisfy

$$
H(x, u, D u) \leq f(x) \quad \text { and } \quad H(x, v, D v) \geq 0 \quad \text { in } \Omega
$$

in the viscosity sense. If $u \leq v$ on $\partial \Omega$, then $u \leq v$ on $\Omega$.

REMARK 1. It is possible to weaken condition (H2) in Lemma 1. Indeed, it is enough to assume the following (H2)' instead of (H2).

(H2) $)^{\prime}$ For each $R>0$ there is a modulus $m_{R}$ such that

$$
H(y, u, \lambda(x-y))-H(x, u, \lambda(x-y)) \leq m_{R}\left(\lambda|x-y|^{2}+|x-y|\right)
$$

for $\lambda \geq 0, x, y \in \Omega$, and $u \in \mathbf{R}$ with $|u| \leq R$.

A more important remark is this: If in addition it is assumed in Theorem 1.1 that $u \in W^{1, \infty}(\Omega)$ or $v \in W^{1, \infty}(\Omega)$, then assumption (H2) can be replaced by the following (H2)".

$(\mathrm{H} 2)^{\prime \prime}$ For each $R>0$ there is a modulus $m_{R}$ such that

$$
H(y, u, \lambda(x-y))-H(x, u, \lambda(x-y)) \leq m_{R}(|x-y|)
$$

for $\lambda \geq 0, x, y \in \Omega$, and $u \in \mathbf{R}$ with $\lambda|x-y| \leq R$ and $|u| \leq R$.

PROOF. If $\sup _{\Omega}(u-v)>0$, then we get a contradiction by following, e.g., the proof of $[1$, Theorem 2.1 or 3 , Theorem 2] because $f<0$ in $\Omega$. Q.E.D.

To continue, we need the following assumptions.

(H3) There is a function $\varphi \in C^{1}(\Omega) \cap C(\bar{\Omega})$ such that

$$
\sup \{H(x, u, D \varphi(x)) \mid x \in \omega, u \in \mathbf{R}\}<0 \text { for all } \omega \subset \subset \Omega .
$$

(H4) The function $p \rightarrow H(x, u, p)$ is convex on $\mathbf{R}^{N}$ for each $(x, u) \in \Omega \times \mathbf{R}$.

THEOREM 1. Assume (H1) - (H4). Let $u, v \in C(\bar{\Omega})$ satisfy

$$
H(x, u, D u) \leq 0 \quad \text { and } \quad H(x, v, D v) \geq 0 \quad \text { in } \Omega
$$

in the viscosity sense. Assume also $u \leq v$ on $\partial \Omega$. Then $u \leq v$ on $\Omega$.

REMARK 2. This is a viscosity solution version of the uniqueness theorem of S. N. Kružkov [9] and is essentially due to S. N. Kružkov (see also [2, 10]). Our proof is, however, simple, direct, and different from that of $[\mathbf{2}, \mathbf{9}, \mathbf{1 0}]$. An example of application of Theorem 1 with $\varphi(x) \not \equiv 0$ is presented in $\S 2$. 
REMARK 3. It is enough to assume the following $(\mathrm{H} 3)^{\prime}$ in place of (H3) in Theorem 1 as the proof below shows.

(H3)' For any $R \in \mathbf{R}$ there is a $\varphi_{R} \in C^{1}(\Omega) \cap C(\bar{\Omega})$ such that

$$
\sup \left\{H\left(x, R, D \varphi_{R}(x)\right) \mid x \in \omega\right\}<0 \quad \text { for all } \omega \subset \subset \Omega \text {. }
$$

If the function $(u, p) \rightarrow H(x, u, p)$ is convex, then (H3) can be replaced in Theorem 1 by

(H3)" There is a function $\varphi \in C^{1}(\Omega) \cap C(\bar{\Omega})$ such that

$$
\sup \{H(x, \varphi(x), D \varphi(x)) \mid x \in \omega\}<0 \text { for all } \omega \subset \subset \Omega .
$$

REMARK 4 . It is possible to replace the convexity assumption (H4) by the next $(\mathrm{H} 4)^{\prime}$ in Theorem 1.

$(\mathrm{H} 4)^{\prime}$ There are functions $\alpha$ and $\beta$ on $(0,1)$ satisfying $\alpha(\theta) \geq 0$ and $\beta(\theta)>0$ for $0<\theta<1$ such that

$$
H(x, u, \theta p+(1-\theta) D \varphi(x)) \leq \alpha(\theta) H(x, u, p)+\beta(\theta) H(x, u, D \varphi(x))
$$

for $x \in \Omega, u \in \mathbf{R}, \theta \in(0,1)$, and $p \in \mathbf{R}^{N}$, where $\varphi$ is from (H3).

For instance, consider the Hamiltonian $H(x, u, p)=h(p)-n(x)$, where $h \in$ $C\left(\mathbf{R}^{N}\right)$ and $n \in C(\bar{\Omega})$. Clearly (H1) and (H2) are satisfied. Assume $n(x)>0$ for $x \in \Omega$ and that $h$ is positively homogeneous of degree $m>0$, i.e., $h(\lambda p)=\lambda^{m} h(p)$ for $\lambda \geq 0$ and $p \in \mathbf{R}^{N}$. Then (H3) and (H4) are satisfied with $\varphi(x) \equiv 0, \alpha(\theta)=\theta^{m}$, and $\beta(\theta)=1-\theta^{m}$.

REMARK 5. Remarks analogous to Remark 1 are valid to Theorem 1.

ProOF. Let $\theta \in(0,1)$, and set

$$
u_{\theta}(x)=\theta u(x)+(1-\theta) \varphi(x) \text { for } x \in \bar{\Omega} .
$$

In view of (H3) we can choose $f \in C(\bar{\Omega})$ such that $H(x, r, D \varphi(x)) \leq f(x)<0$ for $x \in \Omega$ and $r \in \mathbf{R}$. Replacing $\varphi$ by $\varphi-M$ with $M>0$ if necessary, we may assume $\varphi \leq u$ on $\Omega$. Then we see that $u_{\theta} \leq u$ on $\Omega$ and $u_{\theta} \in C(\bar{\Omega})$. A formal calculation reveals that

$$
\begin{aligned}
H\left(x, u_{\theta}, D u_{\theta}\right) & \leq \theta H\left(x, u_{\theta}, D u\right)+(1-\theta) H\left(x, u_{\theta}, D \varphi\right) \\
& \leq \theta H(x, u, D u)+(1-\theta) f(x) \leq(1-\theta) f(x) .
\end{aligned}
$$

Here we have used (H4), (H1) and (1.2). Indeed, it is not hard to see that $H\left(x, u_{\theta}, D u_{\theta}\right) \leq(1-\theta) f(x)$ in $\Omega$ in the viscosity sense. Now Lemma 1 guarantees that $u_{\theta} \leq v$ on $\Omega$ for $0<\theta<1$. Thus we conclude that $u \leq v$ on $\Omega$. Q.E.D.

2. An application. The Dirichlet problem

$$
\begin{cases}a(x) D u \cdot D u-b(x) \cdot D u=0 & \text { in } \Omega, \\ u(x)=g(x) & \text { on } \partial \Omega\end{cases}
$$

arises, e.g. in the study of large deviations or elliptic singular perturbation problems (see L. C. Evans and H. Ishii [4], S. Kamin [8] and references therein). Here $\Omega$ is an open bounded subset of $\mathbf{R}^{N}, a \in C\left(\bar{\Omega} ; M^{N \times N}\right)$, where $M^{N \times N}$ denotes the space of $N \times N$ matrices, the dot "." stands for the Euclidean inner product, $b \in C\left(\bar{\Omega} ; \mathbf{R}^{N}\right)$, and $g \in C(\partial \Omega)$. 
We make the following assumptions:

(A1) There is a constant $\theta>0$ such that

$$
a(x) \xi \cdot \xi \geq|\xi|^{2} \text { for } x \in \Omega \text { and } \xi \in \mathbf{R}^{N} .
$$

(A2) There is a function $\psi \in C^{1}(\bar{\Omega})$ such that

$$
b(x) \cdot D \psi(x) \geq 1 \text { for } x \in \Omega .
$$

Observe that if $u \in C(\bar{\Omega})$ is a viscosity subsolution of (2.1) and (A1) holds, then $u$ satisfies

$$
|D u|^{2} \leq C \text { in } \Omega
$$

in the viscosity sense for some positive constant $C$ (see [2, Theorem I.14]).

THEOREM 2. Under the assumptions above, problem (2.1) has at most one viscosity solution in the class $C(\bar{\Omega})$.

REMARK 6. An assumption like (A2) appears in [8]. The above uniqueness assertion has been proved via the representation formula for the viscosity solution of (2.1) under somewhat stronger regularity assumptions in [4]. In [4] the following assumption is used instead of (A2).

$(\mathrm{A} 2)^{\prime}$ If $x(\cdot) \in H_{\text {loc }}^{1}\left([0, \infty) ; \mathbf{R}^{N}\right)$ and $x(t) \in \bar{\Omega}$ for all $t \geq 0$, then

$$
\int_{0}^{\infty}\left|\frac{d x}{d t}(t)-b(x(t))\right|^{2} d t=\infty .
$$

Note that (A2)' is equivalent to (A2). For the proof of this equivalence we refer the reader to [7].

PROOF. Set

$$
H(x, u, p)=a(x) p \cdot p-b(x) \cdot p \quad \text { for }(x, u, p) \in \Omega \times \mathbf{R} \times \mathbf{R}^{N} .
$$

Clearly $H$ satisfies (H1), (H2)", and (H4). Moreover, if $\varepsilon>0$ is chosen so small that $\varepsilon a(x) D \psi(x) \cdot D \psi(x) \leq \frac{1}{2}$ for all $x \in \Omega$, then we have

$$
\begin{aligned}
H(x, u, \varepsilon D \psi(x)) & =\varepsilon(\varepsilon a(x) D \psi(x) \cdot D \psi(x)-b(x) \cdot D \psi(x)) \\
& \leq-\varepsilon / 2 \text { for } x \in \Omega \text { by (A2). }
\end{aligned}
$$

Therefore (H3) holds for $\varphi=\varepsilon \psi$ with $\varepsilon>0$ sufficiently small. Taking account of Remark 4, we apply Theorem 1.2 to get the conclusion. Q.E.D.

\section{REFERENCES}

1. M. G. Crandall, L. C. Evans, and P. L. Lions, Some properties of viscosity solutions of Hamilton-Jacobi equations, Trans. Amer. Math. Soc. 282 (1984), 487-502.

2. M. G. Crandall and P. L. Lions, Viscosity solutions of Hamilton-Jacobi equations, Trans. Amer. Math. Soc. 277 (1983), 1-42.

3. __ On existence and uniqueness of solutions of Hamilton-Jacobi equations, Nonlinear Anal. (to appear).

4. L. C. Evans and H. Ishii, A PDE approach to some asymptotic problems concerning random differential equations with small noise intensities, Ann. Inst. H. Poincaré. Anal. Non Linéaire 2 (1985), 1-20.

5. H. Ishii, Uniqueness of unbounded viscosity solution of Hamilton-Jacobi equations, Indiana Univ. Math. J. 33 (1984), 722-748. 
6. ___ Existence and uniqueness of solutions of Hamilton-Jacobi equations, Funkcial. Ekvac. 29 (1986), 167-188.

7. H. Ishii and S. Koike, Remarks on elliptic singular perturbation problems (in preparation).

8. S. Kamin, Exponential descent of solutions of elliptic singular perturbation problems, Comm. Partial Differential Equations 9 (1984), 197-213.

9. S. N. Kružkov, Generalized solutions of the Hamilton-Jacobi equations of eikonal type. I, Math. USSR.-Sb. 27 (1975), 406-446.

10. P. L. Lions, Generalized solutions of Hamilton-Jacobi equations, Pitman, Boston, Mass., 1982.

DePARTMENT OF MATHEMATICS, ChUO UNIVERSITY, BUNKYO-KU, TOKYO 112, JAPAN 